\title{
Spectroscopic surveys: a different approach to data reduction
}

\author{
B.Garilli, P.Franzetti, L.Paioro, M.Scodeggio, A.Zanichelli \\ INAF-IASF, Milan \\ via Bassini, 1520133 Milan, Italy \\ bianca@mi.iasf.cnr.it
}

\begin{abstract}
We present VIPGI, an automatized human supervised reduction environment, developed within the VIRMOS project to handle VIMOS guaranteed time data. VIPGI is now offered to the international community to be used on site in Milano and Marseille. Born to handle the highly multiplexed MOS VIMOS data, it has been extended to accomodate also IFU data. The widespread and extensive use of VIPGI has suggested the idea of an integrated environment allowing users not only to reduce, but also to organize data in logical structures, to insert results in a database, and use any user defined plugin for data reduction, analysis and inspection. See http://cosmos.mi.iasf.cnr.it/pandora
\end{abstract}

Keywords: Data Reduction, Astronomical Software, Spectroscopy

\section{INTRODUCTION}

In the last years the number of large telescopes available to the astronomical community has rapidly increased, together with the multiplexing capabilities of the instruments attached to those telescopes. While a normal long-slit spectrograph on a 4-meter class telescope could produce a few tens of spectra per night of observation, today a last generation optical spectrograph can obtain several thousands spectra per night. This productivity increase has rendered obsolete traditional methods of data reduction and analysis, at least as long as these data must be reduced and analyzed in a timely fashion. Moreover it is necessary to develop an efficient and rigorous data organizer and archiver, so that the available data and files would not be lost among hundreds or thousands of similar data and files. General-purpose astronomical software packages like IRAF or MIDAS are not well equipped for these tasks. They offer a huge amount of tasks to manipulate astronomical data which constitute the elementary particles with which, in principle, it could be possible to build up a semi-automatic pipeline. As a matter of facts, however, each step of the reduction process requires the execution of too many such "particles" to allow for a substantial automation. Moreover, such tools do not offer any facility for organizing data, an aspect which must not be neglected: dealing manually with hundreds of spectra per exposure can be a hard job even for the most systematic astronomer.

Among the present day spectrographs, VIMOS is perhaps the most challenging in terms of data production. VIMOS ([3]) is the imaging spectrograph mounted on Unit 3 (Melipal) of the Very Large Telescope (VLT) at the Paranal Observatory. It has been specially designed to be a survey oriented instrument, therefore its multiplexing capabilities have been pushed to the maximum: during a single exposure, up to 800 spectra can be obtained in MOS mode (6400 in IFU mode), and this means a really huge amount of data. Difficulties begin already when trying to find one's way in the jungle of raw science and calibration frames, as distributed via the standard ESO archive procedures, and increase going further along the reduction process, due to the number of calibrations and corrections that are to be applied to the data (at least for spectroscopic ones).

For this reason the VIRMOS Consortium was asked to deliver to ESO all the elements necessary to build an automatic data reduction pipeline. The result of this work is the VIMOS Data Reduction 
Software (DRS). On top of this, to allow the usage of the DRS outside the ESO Data Flow System, we have developed VIPGI (Vimos Interactive Pipeline and Graphical Interface). Based on DRS for the reduction part, VIPGI provides the astronomer with a user-friendly graphical interface for executing the pipeline recipes, with a built-in data organizer, and data browsing and plotting facilities.

Although designed for the reduction of the VIMOS VLT Deep Survey (VVDS) data, VIPGI is sufficiently general to make it able to handle all VIMOS available observing modes and instrument configurations. Its capabilities and the quality of its data reduction pipeline have been verified through the reduction of some 30,000 spectra from the VVDS, demonstrating it can be used to achieve a data reduction accuracy comparable to the one obtained using standard IRAF tasks. Starting November 2003, VIPGI has been offered for usage to the astronomical community, but the scientific validation process has not, so far, covered all VIMOS observing modes, and all VIMOS grisms. Therefore at the moment we require it to be used either in Milano or in Marseille, under the supervision of a VIRMOS Consortium astronomer, who can provide guidance on how to handle non standard situations.

\section{THE VIMOS INTERACTIVE PIPELINE GRAPHICAL INTERFACE}

\subsection{The instrument model}

VIPGI reduction recipes are based onto an instrument model, which analytically describes the main calibration relations required for spectra extraction. The instrument model is separated into three different components: the optical distorsion model (ODM), is used to convert the positions of an observed object on the instrument focal plane to the corresponding position on the CCD frame. The curvature model $(C M)$ is used to trace the edges of the spectrum along the dispersion direction. Finally, the inverse dispersion solution (IDS) is the usual conversion relation between the position in pixels on the CCD and the wavelength coordinate. All these models have been implemented into the code as polynomial relations. Part of the calibration (like e.g. grism characteristics) is provided by tables appended to raw files during the data organization step, while another part (namely first guesses for each polynomial coefficient) is contained into raw files headers. The pipeline uses these first guesses as a starting point for the detailed determination of the polynomials.

\subsection{The Graphical Interface}

Although VIPGI recipes automate to a very large extent the task of reducing VIMOS data, they do not address at all two important and problematic areas of the global data reduction activity. The first one is that of organizing the large volume of VIMOS data in such a way that the correct input is always given to the various recipes, since the recipes do not perform any extensive validation check on their input data. The second one is that of a quick and easy browsing of the data at the various stages of data reduction. It is mainly to address these to points that we have designed VIPGI, the graphical user interface for the VIMOS data reduction pipeline. Such GUI is written almost entirely in Python, to simplify the handling of graphical elements and to take advantage of the object-oriented programming capabilities offered by that language, with only some auxiliary functions devoted to the handling of FITS files carried out by a set of $\mathrm{C}$ programs.

\subsection{Data Organization}

To be used in VIPGI, raw and reduced VIMOS FITS files must be put under the control of VIPGI data organizer. Files are phisically organized into a pre-defined directories structure and renamed following a scheme that extremely simplifies the task of understanding what the content of each file is. Moreover the data organizer provides a logical classification of each file, dividing data according to observation date, instrument mode (MOS, IFU, or Imaging), quadrant, grism or filter used during the observation, type of observation (science exposure, flat field or wavelength calibration lamp, bias frame, and so on), and observation target. These logical categories are then used to simplify the browsing of data, allowing the user to select in a very simple way a specific data category 
of interest, and to ensure that correct input data is provided at all times to the VIPGI reduction recipes.

\subsection{The data reduction pipeline}

VIPGI data reduction is organized in recipes; each one is a stand-alone program that performs one or more reduction steps. All VIPGI data reduction recipes are written entirely in $\mathrm{C}$, to ensure a satisfactory execution speed. When designing recipes, we have tried to group together steps which are normally always executed in the same sequence: for example, bias subtraction, flat field correction and cosmic ray cleaning in the imaging case have been grouped into one recipe. However there is no single "do it all" recipe that can be fed with a bunch of raw data frames to produce completely reduced images or spectra, as we assume that the astronomers will need and want to check at least some of the intermediate data reduction steps. To help astronomers keep the details of the data reduction process under control, the detailed behavior of each recipe can be customized via a set of input parameters, that are stored in a parameter file.

The building blocks for the recipes code are provided by a set of routines and library functions designed to handle VIMOS data. The routines range from the basic "opening a file and reading its content" to spectral extraction, wavelength calibration and 3D data cube reconstruction. General tasks like the handling of FITS files, or of the World Coordinate System, and the detection of stars within imaging exposures (for photometric or astrometric calibration purposes) are carried out by special purpose external software packages (the CFITSIO and WCSTools libraries, and SExtractor, respectively) that have been included within the DRS.

The recipe input and output are always in the form of FITS files, and to avoid increasing the already large number of files produced by VIMOS, the different reduction mid-products (like e.g. 2D extracted, sky-subtracted spectra) together with the various calibration tables useful for the reduction are "appended" as extensions to the original FITS files, instead of creating independent ones. This is done not to save disk space (a multiple extensions file occupies the same amount of bytes as the sum of many single files), but to simplify the astronomer's work, as the data reduction process would otherwise lead to the creation of thousands of files.

For a detailed description of the reduction recipes, we refer to [2] and [5]

\subsection{Quality checks, plotting and analysis}

When starting the reduction of an observation run, the first step to be performed is usually the refinement of the first guesses written into the headers. Small variations in the instrument observing conditions might results in variations of the ODM, CM and IDS coefficients; in this case the first guesses might be not accurate enoguh to let the recipes find and reduce spectra. Graphical tools are provided within VIPGI to inspect and eventually adjust first guesses.

Once first guesses are reasonable for the data at hand, we can proceed with locating spectra on the CCD. A good location of spectra is fundamental to not to increase the noise during the spectral extraction phase. In general, spectra are located within one pixel from the expected position, provided that, for MOS observations, the mask layout designed automatically by the VIMOS Mask Preparation Software (VMMPS, [1]) is not altered by the astronomer by placing manually slits on the mask, thus risking to get spectra which overlap on the CCD. However, instrument flexures and/or badly postioned masks can differentially displace spectra from the theoretical position of a relatively large amount (up to 10 pixels), therefore some check on this step is crucial. To visually check the quality of spectra location, it is possible to display an image where the "edges" of the spectra for each MOS slit or IFU fiber are superimposed.

Once spectra are located, the Inverse Dispersion Solution is computed for each spectrum. The accuracy of the wavelength calibration changes sligthly from grism to grism, but the rms residuals around the best fitting relation typically amount to better than one fifth of a pixel. To visually inspect the goodness of the wavelength calibration, a few plots are available, like e.g. the plot of a single 


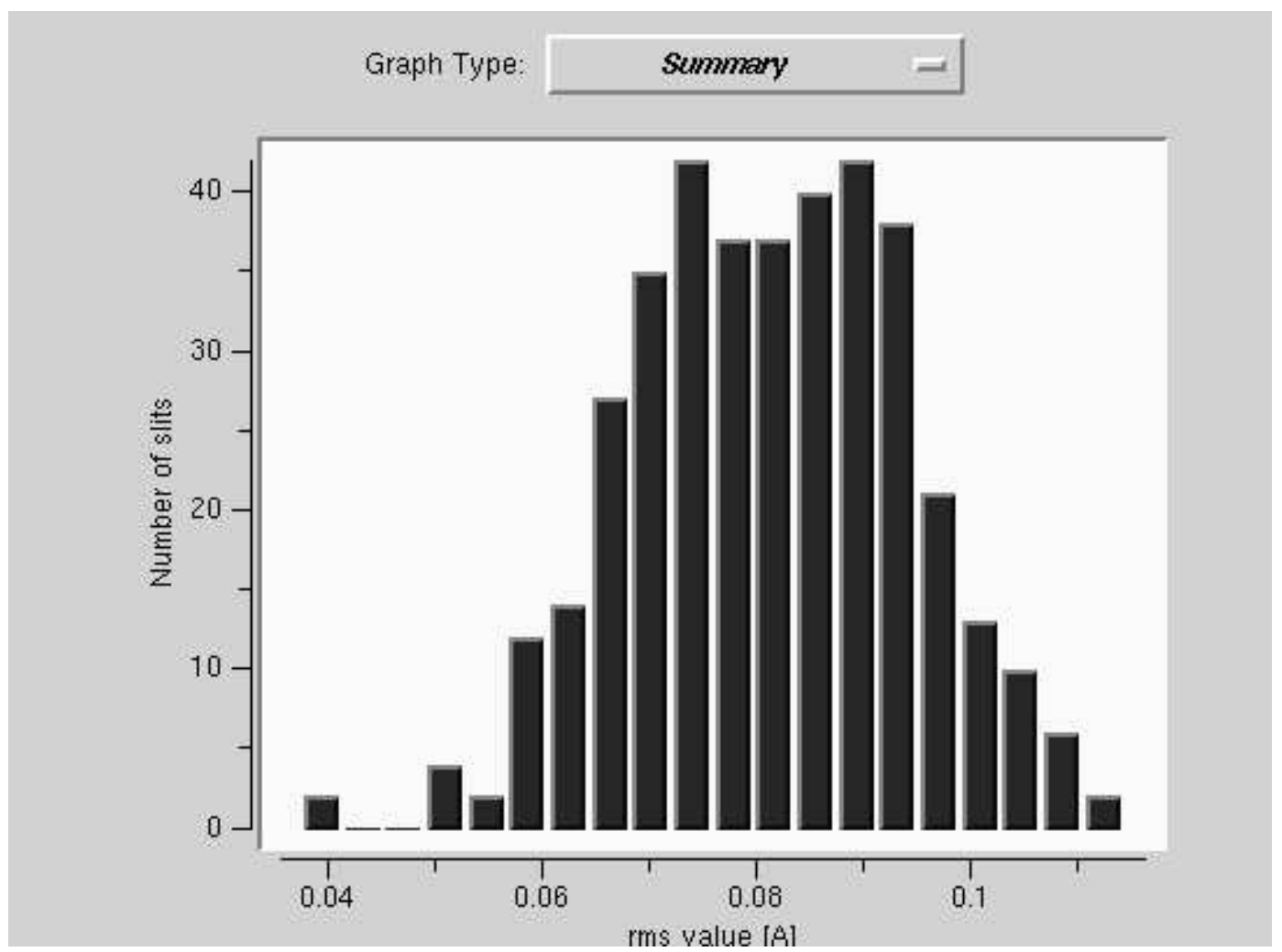

FIGURE 1: Residuals rms histogram plot for an IFU arc lamp exposure

arc line residuals from the best fitting solution for each slit in a MOS mask or each fiber in an IFU observation and an histogram showing the distribution of $r m s$ residual values for all slits in a MOS or all fibers in an IFU exposure. As an example, in figure 1 the residuals rms histogram plot for an IFU arc lamp exposure with the "High Resolution Orange" grism is shown.

A number of different possibilities exist for browsing through one-dimensionally and twodimensionally extracted spectra. Two-dimensional slit spectra for a MOS jitter sequence can be plotted together with all the single-exposures 2D spectra used for the combination, to check the reality of spectral features and the quality of fringing and sky residuals removal.

A tool for plotting and analyzing the extracted one-dimensional spectra is also provided. This tool allows the astronomer to plot each of the one-dimensionally extracted spectra, together with the corresponding two-dimensional and sky 1D spectra. In this way it is possible to check on the reality of spectral features that are present in the one-dimensional spectrum, which could be due to sky subtraction or fringing residuals. Basic plotting interactive tools, like zooming, smoothing, and line fitting are also provided. The astronomer can obtain quick redshift estimates by fitting or marking the position of a set of spectral lines, and using a function that will compute a list of possible redshifts based on a list of known lines in galaxy spectra. Once the user has chosen one possible solution, the expected positions of other lines in the list are marked on the plot, to visually inspect the goodness of the redshift determination.

A "summary" plotting tool is also provided, which incorporates in one display window the functionality of the one-dimensional spectrum, of the lambda calibration, and of the crossdispersion slit profile plotting tools. All functionalities from the one-dimensional plotting tool are preserved. In addition, it is also possible to display information on the astronomical object whose spectrum is being plotted (see figure 2). 


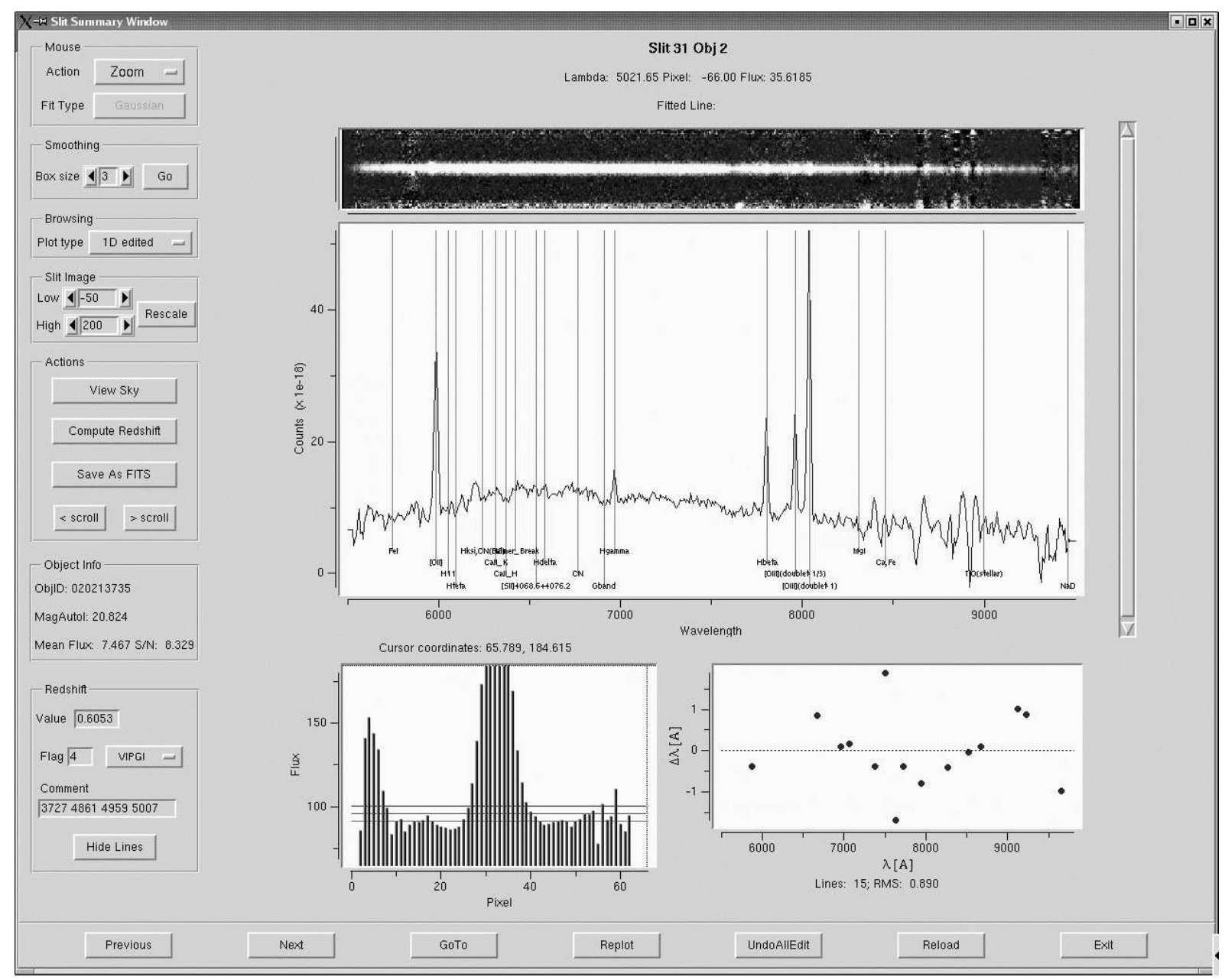

FIGURE 2: The summary plotting tool: from top to bottom, the 2D spectrum, the 1D spectrum, the crossdispersion slit profile(left) and the wavelength calibration rms for the slit (right). On the left column, the funtion buttons and some additional objects information.

\subsection{Overall quality and performances}

To test the quality of the results from our pipeline we have performed parallel reduction of same data with VIPGI and IRAF. This operation has shown that the quality of our spectra, in terms of continuum shape and signal to noise ratio, is basically the same as the one obtained with a manual IRAF reduction. We also have checked our data quality by comparing spectra of objects observed twice during VVDS runs. Checking almost two hundreds spectra we have determined that the global incertainty in redshift measurements on spectra reduced by our pipeline is of the order of $\Delta z \sim 0.001$ (see [4]). An example of extracted 1D spectrum is shown in figure 3

A complete reduction for one quadrant of a single VIMOS MOS or IFU pointing, going from the raw data to the derivation of the wavelength calibration, and to the extraction of one-dimensional spectra, flux and wavelength calibrated and sky subtracted, takes typically around 5 minutes on a Linux PC equipped with an AMD Athlon 2200+ processor and 1 GB of RAM. On the same PC a full reduction for all 4 quadrants of a jitter sequence of 10 MOS or IFU observations can be carried out in approximately 30 minutes (not counting the time eventually dedicated to the visual checks of the data reduction results). The main factor influencing the speed of the data reduction process is the amount of physical memory (RAM) available to the pipeline. On computers with less than 512 MB of RAM VIPGI recipes would require a much longer time to execute. 


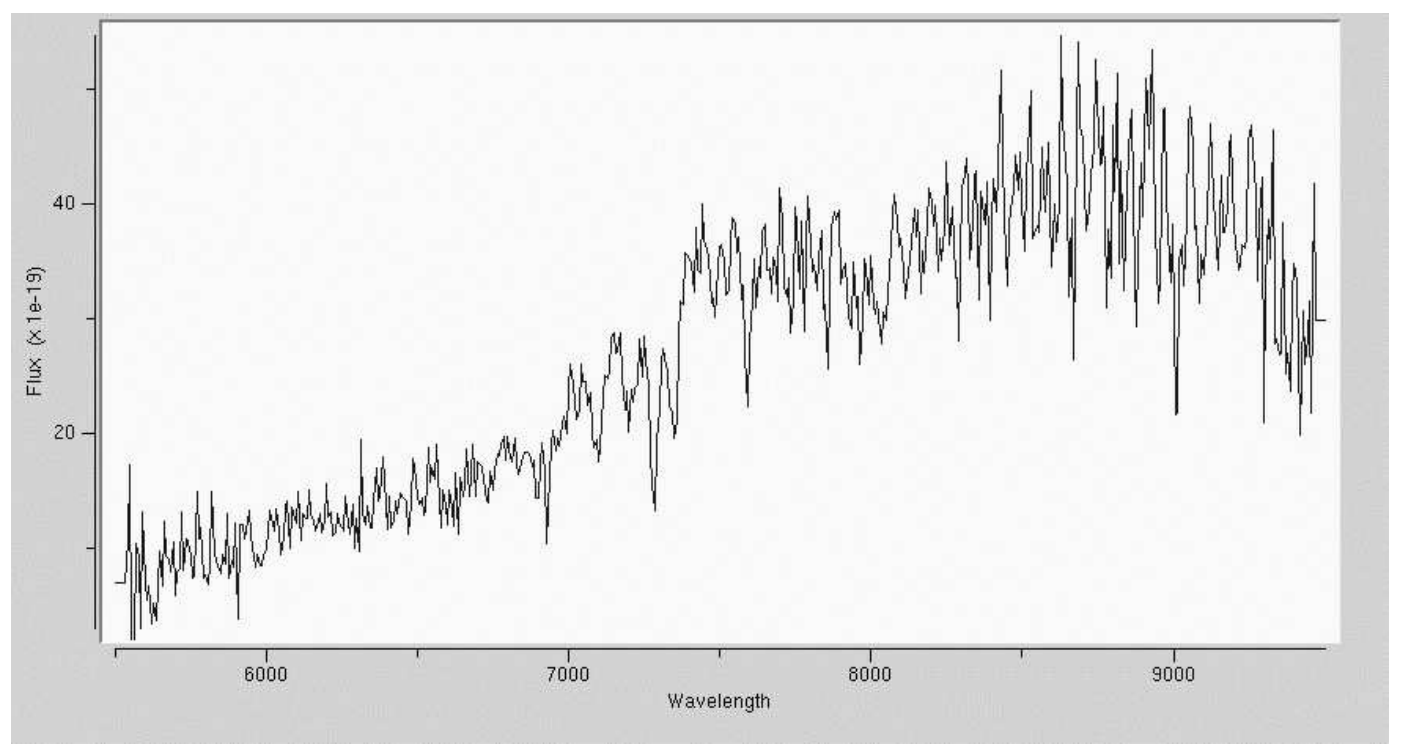

FIGURE 3: $1 \mathrm{D}$ spectrum of a VVDS galaxy at $\mathrm{z}=0.851$. The $\mathrm{Ca} \mathrm{H}$ and $\mathrm{K}$ lines and the Balmer break are clearly visible

\section{PRESENT AND FUTURE DEVELOPMENTS}

After one year of extensive usage, we can safely state that VIPGI has received an excellent feedback from the users, the most appreciated features being the capabilities of data organization, and the possibility to carry out all the reduction AND the first analysis steps within the same environment. On the other hand, the basic limitation of VIPGI is that of being "VIMOS centric": the Data Organizer, but also the plotting and data inspection facilities outlined above, have been deisgned and developed having VIMOS in mind, and it is extremely difficult, if not impossible, to export these tools to be used with other kinds of data.

However, the underlying concept, i.e. an environment allowing Data Organization and handling communications between different analyisis, reduction and display tools, can indeed be exported and developed so that it can be used for any kind of astronomical data, at least in the domain of optical and Near Infrared instruments. This is the basic idea of our new project DRAGO (Data Reduction and Analysis Graphical Organizer). DRAGO has the ambition of providing the user with an easily configurable environment, within which different tools defined by the user can be plugged in.

DRAGO is structured around a powerful data organizer, which provides the user with the facility to select in very simple ways (sub)sets of the available data to work with. The storage and selection functions for the data organizer are handled by either a database server embedded within the package itself, or an external database and/or data archive server, in case the user has already stored and organized his/her data in a database of choice, or is using data from a public data archive. Working in connection with the data organizer there is a series of operational modules that provide the interface to the data reduction and analysis tools. Examples of such modules are the VIMOS data reduction pipeline, the 1-dimensional spectra visualization tool, the Euro3D IFU datacube visualization tool, redshift and spectral line parameter measurement tools.

To fully take advantage of the package functionalities users will have to "import" data into the system, and classify them according to a set of user-defined classification rules, or provide access to already existing database tables.

DRAGO is currently under development, and we refer to Paioro et al, these same proceedings, for a more detailed explanation of its structure and its status 


\section{ACKNOWLEDGEMENTS}

We thank all the VIRMOS consortium for having extensively tested and used VIPGI for reduction, giving us unvaluable suggestions for improvement: we especially thank S.Paltani for pointing out bugs and helping us in their solvimng, and E.Zucca for her precise and puntilious comments. We also thank R.Palsa P. Sartoretti and C.Izzo, from ESO DMD division, for the fruitfull collaboration in developing the DRS basic functions. This work has been partially supported by CNR, COFIN2000, COFIN2003 and INAF.

\section{REFERENCES}

[1] Bottini, D., et al. 2004 A\&A, submitted

[2] Franzetti, P., Scodeggio, M., Garilli, B., Zanichelli, A. et al. (2004), A\&A, submitted

[3] Le Fèvre, O., et al. 2003, The Messenger, 111, 18

[4] Le Fèvre, O., et al. 2004, A\&A, submitted

[5] Zanichelli, A., et al. 2004, submitted 